\title{
Systematic re-evaluation of intraoperative motor-evoked potential suppression in scoliosis surgery
}

Yew Long Lo ${ }^{1,2^{*}}$, Yam Eng Tan ${ }^{3}$, Sitaram Raman ${ }^{3}$, Adeline Teo ${ }^{3}$, Yang Fang Dan ${ }^{3}$ and Chang Ming Guo ${ }^{3}$

\begin{abstract}
Background: Motor- (MEP) and somatosensory-evoked potentials (SSEP) are susceptible to the effects of intraoperative environmental factors.

Methods: Over a 5-year period, 250 patients with adolescent idiopathic scoliosis (AIS) who underwent corrective surgery with IOM were retrospectively analyzed for MEP suppression (MEPS).

Results: Our results show that four distinct groups of MEPS were encountered over the study period. All 12 patients did not sustain any neurological deficits postoperatively. However, comparison of groups 1 and 2 suggests that neither the duration of anesthesia nor speed of surgical or anesthetic intervention were associated with recovery to a level beyond the criteria for MEPS. For group 3, spontaneous MEPS recovery despite the lack of surgical intervention suggests that anesthetic intervention may play a role in this process. However, spontaneous MEPS recovery was also seen in group 4, suggesting that in certain circumstances, both surgical and anesthetic intervention was not required. In addition, neither the duration of time to the first surgical manoeuver nor the duration of surgical manoeuver to MEPS were related to recovery of MEPS. None of the patients had suppression of SSEPs intraoperatively.

Conclusion: This study suggests that in susceptible individuals, MEPS may rarely occur unpredictably, independent of surgical or anesthetic intervention. However, our findings favor anesthetic before surgical intervention as a proposed protocol. Early recognition of MEPS is important to prevent false positives in the course of IOM for spinal surgery.
\end{abstract}

Keywords: Intraoperative monitoring, Motor-evoked potential, Suppression, Amplitude, Scoliosis, Anesthesia

\section{Background}

Motor-evoked potentials (MEPs) are routinely recorded during intraoperative monitoring (IOM) for spinal surgery to ensure integrity of the descending motor tracts. In addition, somatosensory-evoked potentials (SSEP) monitor the ascending dorsal column pathways of the posterior cord. Noteworthy, patients with scoliosis are neurologically intact in general, compared to those undergoing surgery for intramedullary or extramedullary spinal cord disorders.

\footnotetext{
* Correspondence: lo.yew.long@singhealth.com.sg

1 Department of Neurology, National Neuroscience Institute, Singapore

General Hospital, Outram Road, Academia Level 4, Singapore 169608,

Singapore

${ }^{2}$ Duke-NUS Medical School, Singapore, Singapore

Full list of author information is available at the end of the article
}

MEPs and SSEPs are susceptible to the effects of intraoperative environmental factors. For MEPs, volatile anesthetic agents suppress excitability of the motor cortex, resulting in diminished amplitudes [1]. To mitigate the effects of inhalational anesthetics, total intravenous anesthesia (TIVA) has been shown to be effective [2].

SSEPs, in contrast, are less susceptible [3] to the dose-dependent effects of anesthetic agents compared to MEPs. However, blood pressure, anemia, and temperature appear to influence latency and amplitude of responses [4].

Hence, optimal conditions for IOM of spinal surgery include stable blood pressure and core temperature, avoidance of excessive blood loss, use of TIVA to maintain an adequate depth of anesthesia, as well as awareness of the effects of inhalational or neuromuscular blocking agents.

(c) The Author(s). 2018 Open Access This article is distributed under the terms of the Creative Commons Attribution 4.0 International License (http://creativecommons.org/licenses/by/4.0/), which permits unrestricted use, distribution, and 
Gradual suppression of MEPs has been observed in patients under general anesthesia. MacDonald et al. [5] noted abrupt lower limb MEP loss during prolonged scoliosis surgery restored after instrumentation release without deficit and suggested increasing MEP stimulation parameters to offset this effect. Lyon et al. [6] described "anesthetic fade" by virtue of the rate of rise of stimulation voltage threshold proportional to anesthetic duration. The observed effect appears to be more pronounced in myelopathic than neurologically normal patients, but the underlying mechanism responsible remains unclear. In contrast, Holdefer et al. [7] reviewed MEP amplitudes of 50 patients receiving desflurane or propofol during spinal deformity surgery but found no evidence of reducing trend with time. This was corroborated by a separate study [8] which also found no significant MEP amplitude changes over 120 min during propofol anesthesia for spinal surgery. In all, there appears to be conflicting evidence for the occurrence of MEP amplitude reduction intraoperatively and if this phenomenon, if present, is related to anesthetic duration.

We attempt to re-examine these issues relating to motor-evoked potential suppression (MEPS) during IOM in the current study.

\section{Methods}

The institution's ethics committee had previously approved the study protocols.

Over a 5-year period, 250 patients with adolescent idiopathic scoliosis (AIS) who underwent corrective surgery with IOM were retrospectively reviewed. All were previously determined by a neurologist to be without deficits. IOM recordings whereby MEP amplitude reductions beyond $50 \%$ of baseline value at maximum cortical stimulation of $400 \mathrm{~V}$ were defined as MEPS and identified for further analysis. Similarly, IOM recordings whereby SSEP amplitude reduction beyond $50 \%$ of baseline value identified for further analysis. The IOM protocol using TIVA and cortical stimulation methodology have been published previously $[9,10]$.

For induction of anesthesia, propofol at $1-2 \mathrm{mg} / \mathrm{kg}$ and fentanyl at $2 \mu \mathrm{g} / \mathrm{kg}$ was administered. A single administration of $0.8 \mathrm{mg} / \mathrm{kg}$ intravenous atracurium was used to facilitate endotracheal intubation. No further doses of neuromuscular blocking agents were used subsequently. Anesthesia was maintained using the regimen of $10 \mathrm{mg} / \mathrm{kg}$ propofol for the first $10 \mathrm{~min}, 8 \mathrm{mg} / \mathrm{kg}$ for the next $10 \mathrm{~min}$, and $6 \mathrm{mg} / \mathrm{kg}$ for the subsequent length of operation. Fifty percent air in oxygen was administered. Remifentanil at a dose range of $0.03-0.1 \mu \mathrm{g} / \mathrm{kg} / \mathrm{min}$ and morphine were titrated as required for pain relief. Electrocardiography, pulse oximetry, capnography, and direct radial artery pressures were monitored. A bispectral index (BIS) monitor was used in 6 of the patients. All patients were kept normothermic with a warming blanket, and normotensive anesthesia was maintained throughout the operation. Where the BIS monitor was used, the depth of anesthesia was kept to about 40 on the index. As 40 to 60 is considered the range for adequate depth of anesthesia, this was at the deeper end of the range.

After approximately $45 \mathrm{~min}$ post-induction, a train of four-twitch assessment was performed using a nerve stimulator (Fischer Paykel NS242, United Kingdom) on the median nerve at the wrist. Cortical stimulation was commenced only when the amplitude of the fourth twitch (abductor pollicis muscle) was visibly similar to the first, suggesting that the effects of neuromuscular blocking agents have subsided.

Cortical stimulation was delivered by $9-\mathrm{mm}$ gold-plated disc electrodes at C3C4 (International 10-20 system) affixed with collodion. C3 was the active stimulating electrode position for left cortical stimulation, while $\mathrm{C} 4$ was for right cortical stimulation, correspondingly to a cross scalp stimulating configuration. A train of 5 square wave stimuli $0.5 \mathrm{~ms}$ in duration was delivered at $4 \mathrm{~ms}(250 \mathrm{~Hz})$ interstimulus intervals. Stimulation output was increased in steps of $50 \mathrm{~V}$ until a morphologically reproducible MEP with the largest amplitude was elicited. The intensity was then increased and fixed at $10 \%$ above this threshold intensity to obtain a supramaximal MEP response recorded with 13-mm disposable subdermal needles (Technomed Europe, Beek, Netherlands) in the tibialis anterior (TA) bilaterally. Amplifier filter settings were set at $10 \mathrm{~Hz}$ and $2 \mathrm{kHz}$. Input impedance of stimulating and recording electrodes was maintained below $5 \mathrm{k} \Omega$.

MEPs from the TA muscles were recorded bilaterally from the lower limbs by means of a Nicolet Endeavor CR IOM system (Natus Technology, USA). Peak to peak amplitudes and onset latency was measured for MEP responses in each limb, obtained from ipsilateral and contralateral cortical stimulation; ipsilateral MEPs refer to MEPs recorded from the TA on the same side as cortical stimulation. Ten consecutive supramaximal MEPs obtained before insertion of pedicle screws were averaged to obtain a final mean amplitude and latency as a baseline. The baseline sensitivity for signal acquisition was kept at $50 \mu \mathrm{V}$.

Surgical maneuver refers to screw placement or rod placement (Table 1).

Surgical intervention consisted of removal of pedicle screws, brackets, and rods, followed by a wake up test. The decision for wake up tests is left to the surgeon ultimately. No wake up tests were performed for cases 9 to 12 as it was felt that there was sufficient recovery of MEPs without surgical intervention.

Anesthetic intervention referred to the temporary reduction of TIVA and remifentanil to allow for the 
Table 1 Summary of MEPS events for 12 patients with AIS

\begin{tabular}{|c|c|c|c|c|c|c|c|c|c|c|}
\hline Patient & Age & Sex & $\begin{array}{l}\text { Start } \\
\text { anesthesia }\end{array}$ & $\begin{array}{l}\text { Surgical } \\
\text { maneuver }\end{array}$ & $\begin{array}{l}\text { MEP } \\
\text { suppression }\end{array}$ & $\begin{array}{l}\text { Surgical } \\
\text { intervention }\end{array}$ & $\begin{array}{l}\text { Anesthetic } \\
\text { intervention }\end{array}$ & $\begin{array}{l}\text { MEP } \\
\text { recovery }\end{array}$ & $\begin{array}{l}\text { Stage of } \\
\text { operation }\end{array}$ & $\begin{array}{l}\text { Clinical } \\
\text { outcome }\end{array}$ \\
\hline \multicolumn{11}{|l|}{ Group 1} \\
\hline 1 & 17 & $\mathrm{~F}$ & 1319 & 1550 & 1609 & 1618 & 1622 & 1626 & Screw plac & $\mathrm{N}$ \\
\hline 2 & 14 & $\mathrm{~F}$ & 0850 & 0950 & 1001 & 1006 & 1204 & 1246 & Screw plac & $\mathrm{N}$ \\
\hline 3 & 23 & M & 0959 & 1308 & 1346 & 1721 & 1730 & 1800 & Nil & $\mathrm{N}$ \\
\hline 4 & 11 & $\mathrm{~F}$ & 0845 & 1058 & 1101 & 1108 & 1150 & 1214 & Screw plac & $\mathrm{N}$ \\
\hline \multicolumn{11}{|l|}{ Group 2} \\
\hline 5 & 13 & $F$ & 1334 & 1518 & 1534 & 1542 & 1647 & No & Screw plac & $\mathrm{N}$ \\
\hline 6 & 21 & $F$ & 1240 & 1416 & 1423 & 1426 & 1530 & No & Left rod plac & $\mathrm{N}$ \\
\hline 7 & 29 & $F$ & 0919 & 1131 & 1212 & 1258 & 1318 & No & Screw plc & $\mathrm{N}$ \\
\hline 8 & 13 & $\mathrm{~F}$ & 1411 & 1522 & 1535 & 1545 & 1600 & No & Left rod plac & $\mathrm{N}$ \\
\hline \multicolumn{11}{|l|}{ Group 3} \\
\hline 9 & 21 & M & 0908 & 1118 & 1133 & No & 1212 & 1329 & Screw plac & $\mathrm{N}$ \\
\hline 10 & 11 & $F$ & 1025 & 1041 & 1154 & No & 1317 & 1322 & Screw plac & $\mathrm{N}$ \\
\hline 11 & 36 & $\mathrm{~F}$ & 1428 & 1806 & 1811 & No & 1819 & 1849 & Screw plac & $\mathrm{N}$ \\
\hline \multicolumn{11}{|l|}{ Group 4} \\
\hline 12 & 31 & F & 0926 & 1004 & 1045 & No & No & 1112 & Screw plac & $\mathrm{N}$ \\
\hline
\end{tabular}

$M$ male, $F$ female, Plac placement, MEP motor-evoked potential, AIS adolescent idiopathic scoliosis, $N$ normal

reduction in the depth in anesthesia. In 6 cases where the BIS was used, the value rose to around 60 or more indicating a greater probability of deep sleep rather than anesthesia.

MEP amplitude recovery was defined as the recovery MEP amplitude to beyond $50 \%$ of baseline value or latency delay $<10 \%$ of baseline value.

SSEP amplitude recovery is similarly defined as the recovery cortical SSEP (P37) amplitude to beyond $50 \%$ of baseline value or latency delay $<10 \%$ of baseline value.

The following time intervals were noted for each case of possible MEPS:

1. Start of anesthesia to MEPS

2. MEPS to surgical intervention

3. MEPS to anesthetic intervention

4. Start of anesthesia to surgical maneuver

5. Surgical maneuver to MEPS

An anesthetist not involved in the management of each case reviewed intraoperative data to ensure that no confounding factors for MEPS, including vital signs, anesthetic protocol, and interventions, were present.

The Kruskal-Wallis and Mann-Whitney $U$ tests were used to compare interval between groups. A $p$ value $<0.05$ denoted statistical significance.

\section{Results}

A total of 12 AIS patients ( 2 men; mean age 20 (range 11 to 36)) were included in the final analysis. All were clinically well with no transient or permanent neurological deficits after surgical correction.

Based on 250 patients analyzed in this cohort, the estimated frequency of MEPS is $4.8 \%$.

We identified four groups of cases fulfilling MEPS criteria:

1. MEPS with anesthetic and surgical intervention, followed by MEP amplitude recovery

2. MEPS with anesthetic and surgical intervention, without MEP amplitude recovery

3. MEPS with anesthetic but no surgical intervention, followed by MEP amplitude recovery

4. MEPs without anesthetic and surgical intervention, followed by MEP amplitude recovery

Wake up tests performed for cases 1 to 8 all normal.

In group 3 , intraoperative visual monitoring devices such as the O Arm (Medtronic, plc, Colorado, USA) were utilized to provide additional information to the surgical team.

There were no significant differences in time interval 1 between groups 1, 2, and 3 (Kruskal-Wallis test, $H=0.712$, $p>0.05$ ). For time interval 2, comparison between groups 1 and 2 did not reveal statistical significance (Mann-Whitney $U$ test, $Z=0.104, p>0.05$ ). In addition, no statistical difference between groups 1,2 , and 3 was found for time interval 3 (Kruskal-Wallis test, $H=4.348, p>0.05$ ), 4 (Kruskal-Wallis test, $H=0.875, p>0.05$ ), and 5 (KruskalWallis test, $H=1.095, p>0.05$ ). 
Of the 12 cases, all 8 cases with MEP recovery were associated with screw placement. Conversely 2 of 4 cases without MEP recovery (group 2) were associated with rod placement.

The MEP changes of control muscle groups in the upper extremities (first dorsal interossei) did not exceed amplitude reductions beyond $50 \%$ of baseline value at maximum cortical stimulation of $400 \mathrm{~V}$.

No intraoperative SSEP changes were detected for all patients.

Tables 1 and 2 summarize clinical data of the 12 cases analyzed.

\section{Discussion}

Our results show that four distinct groups pf MEPS were encountered over the study period. All 12 patients did not sustain any neurological deficits postoperatively. However, comparing groups 1 and 2 suggest that neither the duration of anesthesia nor speed of surgical or anesthetic intervention were associated with recovery to a level beyond the criteria for MEPS. For group 3, spontaneous MEPS recovery despite the lack of surgical intervention suggests that anesthetic intervention may play a role in this process. However, spontaneous MEPS recovery was also seen in group 4, suggesting that in certain circumstances, both surgical and anesthetic interventions were not required. In addition, comparing groups 1,2 , and 3 suggests that neither the duration of time to the first surgical maneuver nor the duration of surgical manoeuver to MEPS were related to recovery of MEPS.

Table 2 Summary of time intervals for 12 patients with AIS

\begin{tabular}{clllll}
\hline Interval & 1 & 2 & 3 & 4 & 5 \\
\hline Group 1 & & & & & \\
1 & 170 & 9 & 13 & 189 & 19 \\
2 & 71 & 5 & 123 & 60 & 11 \\
3 & 225 & 218 & 224 & 188 & 54 \\
4 & 136 & 7 & 49 & 133 & 3 \\
Group 2 & & & & & \\
1 & 120 & 8 & 73 & 104 & 16 \\
2 & 160 & 3 & 67 & 96 & 7 \\
3 & 173 & 46 & 66 & 131 & 41 \\
4 & 84 & 10 & 25 & 70 & 13 \\
Group 3 & & & & & \\
1 & 145 & & 184 & 130 & 15 \\
2 & 89 & & 172 & 16 & 13 \\
3 & 99 & & 261 & 218 & 5 \\
Group 4 & & & & & \\
1 & 79 & & & 38 & 41 \\
\hline
\end{tabular}

All values shown are in minutes
However, it appears that rod placement (group 2) (Table 1) is associated with more closely with failure of MEP recovery. As the numbers involved are small, it would be difficult to make broad conclusions based purely on these observations.

Overall, this study suggests that in susceptible individuals, MEPS may rarely occur unpredictably, independent of surgical or anesthetic intervention, although the observations made were not on an intentional interference basis. The eventual clinical outcomes were, most importantly, favorable. However, anesthetic intervention was performed in 11 of 12 cases and surgical intervention in 8 of 12 cases. Of note, case 4 in group 1 and cases 9,10 , and 11 in group 3 all had anesthetic intervention and MEP amplitude recovery under BIS monitoring. While the exact reasons remain unclear, the results overall favor anesthetic intervention over surgical intervention. In addition, over the study period, there were no identified cases fulfilling the criteria for significant SSEP changes, suggesting that this form of monitoring may be less vulnerable to interference by external factors intraoperatively. This was seen in contrast to previous reports $[11,12]$.

There is a lack of published information with regard to MEPS. One previous study [6] found the measured voltage threshold needed to produce an MEP of $50 \mu \mathrm{V}$ to be greater at the end of surgery than at baseline. The rate of rise of this threshold was also greater in relation to operating duration and presence of myelopathy. However, direct comparison to our findings may not be valid in view of differences in stimulating and recording parameters, as well as inclusion of neurologically abnormal patients.

To our knowledge, no further published studies have attempted to address MEPS during IOM systematically in a similar manner.

What can we conclude on the physiological basis of MEPS? Several mechanisms have been proposed previously regarding the effect of anesthetic agents on MEPs. These include actions on synaptic transmission, prolongation of axonal refractory period, depressing spinal motor neuron excitability, and facilitation of GABA-mediated inhibitory interneuron actions [13-15]. Our findings are based on elimination of confounding intraoperative factors of blood loss, hypotension, hypothermia, and a standardized anesthetic intervention strategy in neurologically normal patients. In spite, the results do not suggest a durationdependent suppression of cortical excitability for MEPS. Rather, individual factors predisposing to desynchronization of descending volleys summating at spinal interneurons or motor neurons may play a role, but this remains to be further explored.

Hence, we prefer the term MEPS over "anesthetic fade" in view of uncertainty over the purported physiological 
mechanisms, as well as lack of evidence of dose or duration anesthesia plays in AIS patients.

As current studies are limited by small patient numbers, collation of experiences among multiple IOM centers utilizing similar protocols may shed more light on MEPS. Anesthetic depth measurements such as the bispectral index and electroencephalograhy can be incorporated to reduce additional confounders. Monitoring MEPs obtained from an extra muscle group in the lower limb may help mitigate the possibility of recording MEPs with diminished amplitude as a result factor not directly related to neurophysiological dysfunction.

Figure 1 is a flow diagram of a suggested protocol when MEPS is encountered, highlighting the use of anesthetic before surgical intervention based on findings of the current study. Early recognition is important to prevent false positives in the course of IOM for spinal surgery.

Conversely, a recent study examining 62,038 spine surgeries of all categories retrospectively had determined that false negatives occur at a rate of $0.04 \%$ [16]. It would appear that if MEPS occurring at $4.8 \%$ contributes to false positives, then the only patient in group 4 could be considered as a "true" false positive, rendering the overall rate to be $0.4 \%$. Our study, however, consisted of only neurologically normal AIS patients instead of all patients undergoing spinal operations.

For IOM using MEPs overall, it is recommended that interpretation should take into consideration limitations, confounding factors, and the MEP warning criteria be tailored to the type of surgery, as well as the technique and experience of the monitoring team [17]. To date, disappearance of the recorded MEP signals is the main warning criterion yet proposed for spinal cord monitoring. This is based on variability that challenges other criteria, high sensitivity to central motor disturbances, the likelihood that pathophysiology will affect many corticospinal axons because the tract is very small in the spinal cord, and the rapid failure of ischemic lower motor neurons [18-20].

\section{Conclusions}

This study suggests that in susceptible individuals, MEPS may rarely occur unpredictably, independent of surgical or anesthetic intervention. However, our findings favor anesthetic before surgical intervention as a proposed protocol. Early recognition of MEPS is important to prevent false positives in the course of IOM for spinal surgery.

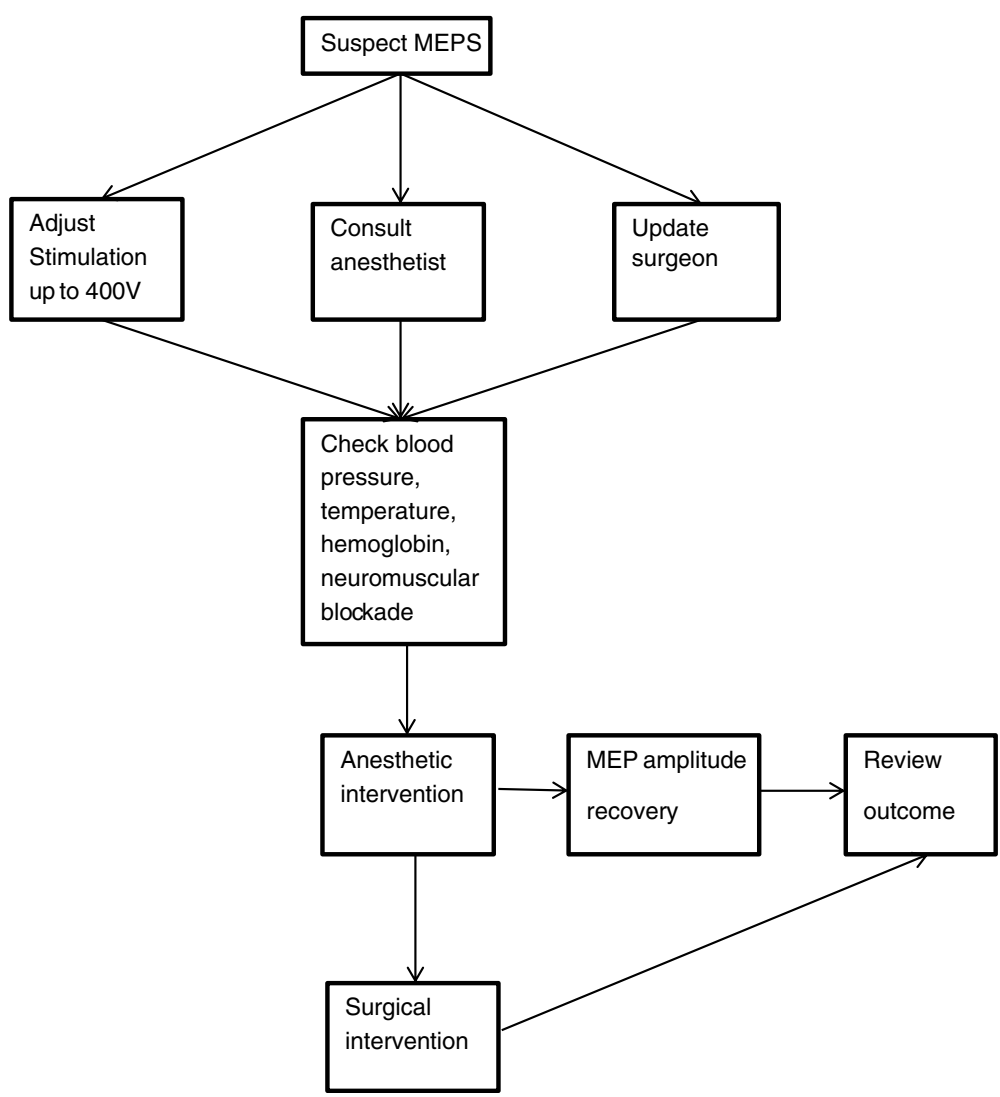

Fig. 1 Flow diagram of a suggested protocol of action for MEPS in scoliosis surgery 


\section{Abbreviations}

BIS: Bispectral index; IOM: Intraoperative monitoring; MEP: Motor-evoked potential; MEPS: Motor-evoked potential suppression; SEP: Somatosensoryevoked potential; TA: Tibialis anterior; TIVA: Total intravenous anesthesia

\section{Authors' contributions}

YLL wrote the paper and conceptualized the study. YET and AT analyzed the data. SR contributed to the data collection and paper writing. YFD monitored and collected the data. GCM operated and conceptualized the study. All authors have read and approved the manuscript.

\section{Ethics approval and consent to participate}

Ethical committee of the Singapore General Hospital has previously approved the study. Consent to participate was obtained from patients even as the data obtained were for clinical service.

\section{Consent for publication}

Written informed consent was obtained from the patients for publication of individual details and images in the manuscript. The consent form is held in the patients' clinical notes.

\section{Competing interests}

The authors declare that they have no competing interests.

\section{Publisher's Note}

Springer Nature remains neutral with regard to jurisdictional claims in published maps and institutional affiliations.

\section{Author details}

Department of Neurology, National Neuroscience Institute, Singapore General Hospital, Outram Road, Academia Level 4, Singapore 169608, Singapore. ${ }^{2}$ Duke-NUS Medical School, Singapore, Singapore. ${ }^{3}$ Singapore General Hospital, Singapore, Singapore.

Received: 16 March 2018 Accepted: 12 June 2018

Published online: 02 July 2018

\section{References}

1. Calancie B, Klose KJ, Baier S, et al. Isoflurane-induced attenuation of motor evoked potentials caused by electrical motor cortex stimulation during surgery. J Neurosurg. 1991;74:897-904.

2. Liu EH, Wong HK, Chia CP, Lim HJ, Chen ZY, Lee TL. Effects of isoflurane and propofol on cortical somatosensory evoked potentials during comparable depth of anesthesia as guided by bispectral index. Br J Anaesth. 2005:94(2):193-7.

3. Freye E, Hartung E, Schenk GK. Somatosensory-evoked potentials during block of surgical stimulation with propofol. Br J Anaesth. 1989:63:357-9.

4. Nuwer M. Spinal cord monitoring. In: Evoked potential monitoring in the operating room. New York: Raven Press; 1986. p. 49-101.

5. MacDonald DB, Al Zayed Z, Khoudeir I, Stigsby B. Monitoring scoliosis surgery with combined multiple pulse transcranial electric motor and cortical somatosensory-evoked potentials from the lower and upper extremities. Spine (Phila Pa 1976). 2003;28:194-203.

6. Lyon R, Feiner J, Lieberman JA. Progressive suppression of motor evoked potentials during general anesthesia: the phenomenon of "anesthetic fade". J Neurosurg Anesthesiol. 2005;17:13-9.

7. Holdefer RN, Anderson C, Furman M, Sangare Y, Slimp JC. A comparison of the effects of desflurane versus propofol on transcranial motor-evoked potentials in pediatric patients. Childs Nerv Syst. 2014;30:2103-8.

8. Liu HY, Zeng HY, Cheng H, Wang MR, Qiao H, Han RQ. Comparison of the effects of etomidate and propofol combined with remifentanil and guided by comparable BIS on transcranial electrical motor-evoked potentials during spinal surgery. J Neurosurg Anesthesiol. 2012;24:133-8.

9. Lo Y, Dan Y, Tan Y, Teo A, Tan S, Yue W, Guo C, Fook-Chong S. Clinical and physiological effects of transcranial electrical stimulation position on motor evoked potentials in scoliosis surgery. Scoliosis. 2010;23(5):3.

10. Lo YL, Dan YF, Tan YE, Nurjannah S, Tan SB, Tan CT, Raman S. Intraoperative motor-evoked potential monitoring in scoliosis surgery: comparison of desflurane/nitrous oxide with propofol total intravenous anesthetic regimens. J Neurosurg Anesthesiol. 2006;18(3):211-4.
11. Kalkman CJ, ten Brink SA, Been HD, Bovill JG. Variability of somatosensory cortical evoked potentials during spinal surgery. Effects of anesthetic technique and high-pass digital filtering. Spine (Phila Pa 1976). 1991;16:924-9.

12. Maurette P, Simeon F, Castagnera L, Esposito J, Macouillard G, Heraut LA. Propofol anaesthesia alters somatosensory evoked cortical potentials. Anaesthesia. 1988;43(S):44-5.

13. Woodforth IJ, Hicks RG, Crawford MR, Stephen JP, Burke D. Depression of I waves in corticospinal volleys by sevoflurane, thiopental, and propofol. Anesth Analg. 1999;89:1182-7.

14. Antkowiak B. Different actions of general anesthetics on the firing patterns of neocortical neurons mediated by the GABA(A) receptor. Anesthesiology. 1999;91:500-11

15. Kakinohana M, Fuchigami T, Nakamura S, Kawabata T, Sugahara K. Propofol reduces spinal motor neuron excitability in humans. Anesth Analg. 2002:94:1586-8.

16. Tamkus AA, Rice KS, McCaffrey MT. Perils of intraoperative neurophysiological monitoring: analysis of "false-negative" results in spine surgeries. Spine J. 2018;18:276-84

17. Macdonald DB, Skinner S, Shils J, Yingling C. American Society of Neurophysiological Monitoring. Intraoperative motor evoked potential monitoring - a position statement by the American Society of Neurophysiological Monitoring. Clin Neurophysiol. 2013;124:2291-316.

18. MacDonald DB, Janusz M. An approach to intraoperative neurophysiologic monitoring of thoracoabdominal aneurysm surgery. J Clin Neurophysiol. 2002;19:43-54

19. Calancie B, Molano MR. Alarm criteria for motor-evoked potentials: what's wrong with the "presence-or-absence" approach? Spine. 2008;33:406-14.

20. Macdonald DB, Al Zayed Z, Al Saddigi A. Four-limb muscle motor evoked potential and optimized somatosensory evoked potential monitoring with decussation assessment: results in 206 thoracolumbar spine surgeries. Eur Spine J. 2007:16(Suppl 2):S171-87.

\section{Ready to submit your research? Choose BMC and benefit from:}

- fast, convenient online submission

- thorough peer review by experienced researchers in your field

- rapid publication on acceptance

- support for research data, including large and complex data types

- gold Open Access which fosters wider collaboration and increased citations

- maximum visibility for your research: over $100 \mathrm{M}$ website views per year

At BMC, research is always in progress.

Learn more biomedcentral.com/submissions 